\title{
STI
}

\section{Barriers and facilitators to human papillomavirus vaccination among Chinese adolescent girls in Hong Kong: a qualitativequantitative study}

T T C Kwan, K K L Chan, A M W Yip, K F Tam, A N Y Cheung, P M C Young, P W H Lee and H Y S Ngan

Sex Transm Inf 2008;84;227-232; originally published online 6 Feb 2008;

doi:10.1136/sti.2007.029363

Updated information and services can be found at:

http://sti.bmj.com/cgi/content/full/84/3/227

\section{These include:}

References This article cites 10 articles, 2 of which can be accessed free at: http://sti.bmj.com/cgi/content/full/84/3/227\#BIBL

\section{Rapid responses You can respond to this article at:} http://sti.bmj.com/cgi/eletter-submit/84/3/227

Email alerting Receive free email alerts when new articles cite this article - sign up in the box at service the top right corner of the article

Notes

To order reprints of this article go to:

http://journals.bmj.com/cgi/reprintform

To subscribe to Sexually Transmitted Infections go to:

http://journals.bmj.com/subscriptions/ 


\title{
Barriers and facilitators to human papillomavirus vaccination among Chinese adolescent girls in Hong Kong: a qualitative-quantitative study
}

\author{
T T C Kwan, ${ }^{1}$ K K L Chan, ${ }^{1}$ A M W Yip,, ${ }^{1}$ K F Tam, ${ }^{1}$ A N Y Cheung, ${ }^{2}$ P M C Young, ${ }^{3}$ \\ P W H Lee, ${ }^{4}$ H Y S Ngan ${ }^{1}$
}

${ }^{1}$ Division of Gynaecological Oncology, Department of Obstetrics and Gynaecology, University of Hong Kong, Hong Kong, China; ${ }^{2}$ Department of Pathology, University of Hong Kong, Hong Kong, China; ${ }^{3}$ School of Nursing, Hong Kong Polytechnic University, Hong Kong, China; ${ }^{4}$ Division of Clinical Health Psychology, Department of Psychiatry, University of Hong Kong, Hong Kong, China

Correspondence to: Professor H Y S Ngan Department of Obstetrics and Gynaecology, 6/F, Professorial Block, Queen Mary Hospital, Pokfulam, Hong Kong SAR,

China; hysngan@hkusua.hku.hk

Accepted 23 January 2008

Published Online First

6 February 2008

\begin{abstract}
Objectives: To explore perceptions towards cervical cancer, human papillomavirus (HPV) infection and HPV vaccination and to identify factors affecting the acceptability of HPV vaccination among Chinese adolescent girls in Hong Kong.
\end{abstract}

Methods: Six focus groups were conducted with Chinese adolescent girls (median age 16 years, age range 13-20, $\mathrm{n}=64$ ) in Hong Kong in April 2007. Thematic analysis was employed to identify major themes related to cervical cancer and HPV vaccination. A supplementary questionnaire was administered to all participants before and after group discussion to assess their knowledge, attitudes and intention to be vaccinated and to collect demographic information.

Results: Participants' knowledge on cervical cancer was limited and HPV was largely unheard of. They had difficulty understanding the mechanism linking cervical cancer with HPV infection. Participants held a favourable attitude towards HPV vaccination but the perceived timing of vaccination varied. Barriers to vaccination include high monetary cost, uncertain length of vaccine effectiveness, low perceived risk of HPV infection, no immediate perceived need of vaccination, anticipated family disapproval and fear of the pain of injection. Factors conducive to vaccination include perceived family and peer support and medical reassurance on safety and efficacy of vaccine. The differences on knowledge, attitudes, intention to be vaccinated now and willingness to conform to significant others before and after the discussion were statistically significant, with an increased tendency towards favouring vaccination after the focus group.

Conclusions: Participants favoured HPV vaccination despite not feeling an immediate need to be vaccinated. Interventions could focus on providing professional information on HPV vaccination and raising adolescents' perceived need to take preventive measures against HPV infection.

Cervical cancer is a serious global disease afflicting many women each year. It is the fifth most common female cancer in Hong Kong, with an age-standardised incidence rate of 9.5 per 100000 women. ${ }^{1}$ Human papillomavirus (HPV) types 16 and 18 , the strains accounting for nearly $70 \%$ of cervical cancers worldwide, ${ }^{2}$ were among the most common high-risk HPV identified in Hong Kong women. ${ }^{3}$ The introduction of vaccines against HPV 16 and 18 can potentially lower the local cervical cancer incidence. Coverage is crucial, however, and vaccination acceptability in the target population can greatly affect coverage. Despite adolescents being the ideal target for HPV vaccination, their views on the issue are little known. In Asia, adolescents have a comparatively conservative cultural upbringing. The mean age of first sex in Hong Kong was 18.9 years (1996 data), which was relatively late in comparison with other western countries. ${ }^{4}$ Overall HPV prevalence is, however, projected to rise as a result of increased sexual openness and permissiveness among Hong Kong adolescents. ${ }^{5}$ Data on the adolescents' perceptions of HPV vaccination are practically non-existent. The objectives of this study were to explore adolescent girls' perceptions towards cervical cancer, HPV infection and HPV vaccination, and factors affecting their acceptability of HPV vaccination. Our findings would be useful in identifying the need for HPV vaccination promotion among young Chinese in Hong Kong, as well as those in other parts of China and neighbouring Asian countries that share a similar oriental cultural heritage.

\section{METHODS}

\section{Subjects}

Healthy ethnic Chinese adolescent girls were recruited from a community youth centre and a secondary school using purposive sampling. Adolescents attending the youth centre for recreational activities were invited to join the study by the centre staff. In the participating school, form 2 and form 7 students (equivalent to UK form 2 and lower 6th, respectively) were invited to participate by their teachers. Intending participants were given an information sheet about the study. A signed parental consent was also obtained from all participants.

\section{Data collection}

Qualitative research methods were deemed most appropriate in exploring attitudes and beliefs that are not previously or systematically known. In this study, a qualitative-quantitative approach was used in that focus group discussions were conducted supplemented by a 27-item questionnaire administered before and after the focus group to detect changes. Groups of 10-12 girls were formed according to age or educational level to increase within-group homogeneity. Participants were assured of the confidentiality and anonymity of all data collected to encourage an open expression of opinions. Each discussion lasted approximately 
Box 1: Standardised information given to the participants during the focus group

1. Cervical cancer is a prevalent female cancer. Each year there are over 400 new cases in Hong Kong.

2. Cervical cancer mostly affects middle-aged or elderly women. Recent trends show that the risk in younger women has, however, increased slightly.

3. The chance of having cervical cancer is minimal in women with no sexual experience.

4. Having multiple sexual partners increases the risk of cervical cancer.

5. Other high-risk factors include smoking, sex at an early age, long-term use of oral contraceptives and high parity.

6. Cervical cancer screening can effectively prevent cervical cancer.

7. Sexually experienced women aged 25 years or above should receive regular cervical cancer screening. A woman is screened annually for the first 2 years. If results are normal for both years, she can then be screened every 3 years afterwards.

8. There are many subtypes of HPV with various modes of transmission. Different subtypes cause different diseases such as skin diseases and genital diseases.

9. Some HPV are called high-risk HPV because they can cause cancer. These HPV are mainly sexually transmitted. Most people who become infected with high-risk HPV will not have symptoms and most will clear the infections on their own.

10. All women who have had sex may be at risk of being infected by high-risk HPV.

11. Most HPV infections will disappear on their own.

12. Cervical cancer may occur if the cells in the cervix are persistently infected by high-risk HPV.

13. Vaccination helps to raise the body's ability to fight against infections.

14. HPV vaccination can prevent cervical cancer.

15. HPV vaccination raises the body's immunity against highrisk HPV. As a result, the risk of cervical cells being infected and subsequently developing into cervical cancer is lowered.

16. The best time for HPV vaccination is before any experience of sexual contact.

17. Based on research findings, the vaccines have been proved effective for at least 5 years. They are generally safe.

Common side effects include soreness and swelling at the injection site, fever and headache. These side effects are usually mild in nature and short-lived. The cost of vaccination is approximately \$HK3000 (GBf187).

18. The vaccines are not $100 \%$ effective against cervical cancer. Regular cervical cancer screening is necessary after HPV vaccination.

one and a half hours and was led by two facilitators in the field of gynaecological oncology or clinical health psychology. A discussion guide was developed by the research team to facilitate the flow of discussion. Based on findings from other studies, the participants were expected to have little knowledge about HPV infection and vaccination. ${ }^{67}$ Therefore, standardised health information was provided intermittently as related to the topic being discussed at the time (box 1). Information was given after participants had shared their views or when they expressed little knowledge or misconceptions on a specific issue.

The self-administered questionnaire was designed to assess: (1) knowledge about cervical cancer and HPV infection; (2) attitudes towards HPV vaccination measured by participants' beliefs about the consequences of being vaccinated now; (3) perceived support from family and friends; (4) intention to be vaccinated and (5) willingness to conform to others' decisions. Sample questions are listed in box 2. Data on demographics were also collected.

Travel reimbursement in the amount of $\$ H K 100$ (or GB£6.25) was given to each participant.

Box 2: Sample items from the questionnaire assessing participants' knowledge on cervical cancer and HPV infection and their attitudes towards HPV vaccination

- Knowledge on cervical cancer and HPV infection-15 items (True/False/Don't know answers). 1 score for each correct answer and 0 score for each incorrect or "Don't know" answer.* Examples:

- Cervical cancer is a disease mostly in older women, say middle age or above.

- Cervical screening can effectively prevent cervical cancer.

- Having multiple sexual partners increases the chance of getting cervical cancer.

- HPV infection causes cervical cancer.

- Anyone who has had sex before is at risk of HPV infection.

- There is a vaccine to prevent HPV infection.

- Attitudes and perceived support from significant others towards HPV vaccination-9 items measured by 4-point Likert scale. $^{*}$ Examples:

- Do you think having this vaccine now is: bad, 1/ somewhat bad, 2/somewhat good, 3/good, 4.

- Do you think having this vaccine now is: unnecessary, $1 /$ somewhat unnecessary, 2/somewhat necessary, 3/ necessary, 4.

- Do you think having this vaccine now is: harmful, 1/ somewhat harmful, 2/somewhat beneficial, 3/beneficial, 4.

- Do you think your family thinks you should have this vaccine: definitely no, 1/no, 2/yes, 3/definitely yes, 4 .

- Intention to be vaccinated-1 item.

- If this vaccine is available for you now, how likely is it that you will have it: very unlikely/unlikely/likely/very likely.

- Willingness to conform to friends' and family's decisions-2 items.

- Imagine, some of your best friends have already had a vaccination to prevent cervical cancer, how willing are you also to have it: very unwilling/unwilling/willing/very willing.

- Imagine, your mother or a close female family member decides to take you to the doctor's clinic for a vaccination to prevent cervical cancer. How willing are you to comply: very unwilling/unwilling/willing/very willing.

*The sum of all items in the section was obtained, with a higher score indicating more knowledge or more positive attitude. 


\section{Analyses}

All focus group discussions were audiotaped and transcribed verbatim. Data were sorted and organised. Thematic analysis was used to code and categorise the data. ${ }^{9}$ Central and repetitive themes were highlighted and conceptual similarities across themes were noted..$^{10}$ Data were compared across groups with the aim of identifying differences as well as generating the most representative summative categories. Findings were reviewed by other research team members and core themes were highlighted according to a consensus view. Conclusions formed are regarded as verifiable with a clear data trail and are backed up by a sufficiently robust dataset. At this stage, however, the representativeness of the data is not explored or assumed.

Data from the questionnaire were analyzed by Spearman's rho correlations to explore associations among different measures. Before and after focus group scores on knowledge and attitudes were compared by Student's paired t-tests. Wilcoxon signed ranks tests were used to detect changes in vaccination intention and willingness to conform. Data analysis was performed using SPSS version 15.0 (SPSS Inc, Chicago, Illinois, USA). A p value of less than 0.05 was considered statistically significant.

\section{RESULTS}

\section{Participants' background and demographics}

Sixty-four girls were recruited into six focus groups. There were two groups of girls aged 13-14 years (A) ( $n=20)$, three groups aged $14-17$ years $(B)(n=34)$ and one group aged $17-20$ years (C) $(n=10)$. Participants were grouped primarily by age although a few girls were grouped to stay in the same group with their peers upon their request. As a result, the age groups overlapped. Participant characteristics are summarised in table 1. When participants were asked about their "acceptable" age for sex, 18 years old or above was deemed appropriate by most of them.

Table 1 Participant characteristics $(n=64)$

\begin{tabular}{|c|c|}
\hline Characteristics & $\begin{array}{l}n(\%) \text { unless } \\
\text { otherwise stated }\end{array}$ \\
\hline Age in years, median (range) & $16(13-20)$ \\
\hline \multicolumn{2}{|l|}{ Parental marital status, n (\%) } \\
\hline Married & $51(80)$ \\
\hline Divorced/separated & $6(9)$ \\
\hline Cohabiting & $5(8)$ \\
\hline Widowed & $2(3)$ \\
\hline \multicolumn{2}{|l|}{ Parental education, n (\%) } \\
\hline Primary & $18(28)$ \\
\hline Secondary & $41(64)$ \\
\hline Tertiary & $5(8)$ \\
\hline \multicolumn{2}{|c|}{ Monthly family income, in $\$ \mathrm{HK}^{*}(\%)$} \\
\hline On government assistance & $3(5)$ \\
\hline$\$ 10000$ and below & $11(17)$ \\
\hline$\$ 10001-15000$ & $18(28)$ \\
\hline$\$ 15001-30000$ & $23(36)$ \\
\hline$\$ 30001$ and above & $6(9)$ \\
\hline Missing data & $3(5)$ \\
\hline \multicolumn{2}{|c|}{$\begin{array}{l}\text { Past experience with physical intimacy (participants may choose } \\
\text { more than one answer), } \mathrm{n}(\%)\end{array}$} \\
\hline Holding hands—yes & $42(66)$ \\
\hline Embracing-yes & $34(53)$ \\
\hline Kissing-yes & $23(36)$ \\
\hline Caressing-yes & $7(11)$ \\
\hline Sexual intercourse-yes & $5(8)$ \\
\hline
\end{tabular}

${ }^{*}$ GBf 1 = \$HK16.01.

\section{Cervical cancer and HPV infection}

All participants had heard of cervical cancer from the mass media. Cervical cancer was regarded as a common disease of grown-up women, which was of little concern to the participants. Multiple probable causes of cervical cancer were suggested by the participants, including lifestyle factors (eg, unhealthy diet and poor personal hygiene), increased age, heredity, environmental pollutants, stress and infection. Sexual practices were regarded as being relevant, with particular reference to promiscuity, having multiple sexual partners and a high frequency of sex as risk factors. None of the participants had, however, any realistic understanding of the mechanism linking sex with cervical cancer.

No participant had any knowledge about HPV. A few girls had heard of the lay term for genital warts. All they knew of genital warts was that they were related to sex. Participants had initial difficulties understanding that different HPV substrains had different modes of transmission leading to different diseases and the role of high-risk HPV in the development of cervical cancer. Repeated clarifications by the facilitators were required. On being told of the sexually transmitted nature of high-risk $\mathrm{HPV}$, the overwhelming response was a raised alertness and caution towards sex. Many participants indicated that they would think twice before having sex for fear of infection. One participant in group C said "It (HPV) made the consequence of sex more serious", whereas another in group A said "I would only consider (sex) if he is worth it". Participants were surprised while at the same time relieved to learn of the asymptomatic and self-healing nature of most high-risk HPV infections. One girl in group C asked "So, it (HPV) is not so scary after all... why is it called high-risk?"

\section{HPV vaccination}

Over half of the participants had heard of vaccination against cervical cancer from television but no one could provide any details about the vaccines nor the mode of action involved. When asked for whom the vaccine was intended, the participants could not clearly differentiate between the candidates for cervical cancer screening with those for vaccination. Some girls noted that those who frequently had sex needed it. There were many arguments on whether the vaccine should be given before or after having sex-reflecting confusion between a prophylactic vaccine and a therapeutic one. Explanation on the prophylactic nature of the current HPV vaccines had to be given. Rather dubiously, one girl in group A claimed to have received the vaccine at school and thus noted (erroneously) that she no longer needed to attend cervical cancer screening in future. The limitations of the current HPV vaccines and the need to continue screening after vaccination were stressed by the facilitators afterwards.

Questions were raised by the participants concerning the vaccines' costs, potential discomfort, adverse effects (in particular on fertility and appearance), the number of injections required and duration of effectiveness. Before the issue of cost was mentioned, the majority of the girls chose to be vaccinated on condition that authoritative reassurances about safety and efficacy were given. Those who refused provided reasons including fear of pain, "still too young", no immediate perceived need, no plan to have sex in the near future and the uncertain duration of effectiveness of the vaccines. On being told that the current data on effectiveness was available up to 5 years, most participants regarded it as being "not long enough". After the cost of vaccination was disclosed, the entire picture on acceptability was altered. Many girls who previously expressed 
Table 2 Correlations between knowledge, attitudes, perceived support of significant others and HPV vaccination measured after focus group

\begin{tabular}{lllll}
\hline & $\begin{array}{l}\text { Knowledge about } \\
\text { cervical cancer } \\
\text { and HPV infection }\end{array}$ & $\begin{array}{l}\text { Attitudes } \\
\text { towards HPV } \\
\text { vaccination }\end{array}$ & $\begin{array}{l}\text { Perceived family } \\
\text { support towards } \\
\text { vaccination }\end{array}$ & $\begin{array}{l}\text { Perceived peer } \\
\text { support towards } \\
\text { vaccination }\end{array}$ \\
\hline Intention to be vaccinated now & -0.170 & $\boldsymbol{r}_{\boldsymbol{s}}^{\dagger}$ & $\boldsymbol{r}_{\boldsymbol{s}}$ & $\boldsymbol{r}_{\boldsymbol{s}}$ \\
Willingness to conform to family's decision & -0.127 & $0.448 \%$ & $0.555 \%$ & $0.349 \ddagger$ \\
Willingness to conform to peers' decision & -0.202 & 0.208 & $0.316^{*}$ & $\mathrm{NA}$ \\
\hline
\end{tabular}

HPV, human papillomavirus; NA, not applicable.

${ }^{*} \mathrm{p} \leqslant 0.05 . \dagger r_{s}$, Spearman rank correlation coefficient. $\mathrm{t} p \leqslant 0.01$.

a willingness to be vaccinated opted out. One girl in group C stated "Where will I get the money for it (HPV vaccine)? At this price, my family could not support me either". Another girl in group A summarised the sentiments well, noting "I am too young for the vaccine. If it is good only for 5 years, it will be a waste of money to take it now because I am not going to start sex in the next few years. I will wait till I am 18 years or older".

With regard to the perceived family reaction to the vaccine, some believed that family members would be supportive for "it is just a protection". Others anticipated family disapproval because of their young age. One girl in group C said "They will think that I am too young for sex and have no need for this vaccine now". There were also concerns expressed about how others might see them if people knew that the vaccine was for the prevention of a sexually transmitted infection. "If my family knows what this (HPV vaccine) is for and if I say I want it, they would think that I am fooling around", said a girl in group $B$.

\section{Findings on knowledge, attitude and vaccination intention}

Data obtained from the questionnaire are presented in table 2. Attitudes and perceived support from family or peers correlated significantly with the intention to be vaccinated and willingness to conform. Mean scores of knowledge and attitudes, the proportions of individuals having a positive intention to be vaccinated and of those who were willing to conform to significant others increased significantly after focus group discussion (table 3). A summary of the findings are presented in box 3 .

\section{DISCUSSION}

The participants' knowledge about cervical cancer was grossly inadequate, whereas HPV was unheard of. The teenage girls had difficulty understanding the nature of high-risk HPV infections and the mechanisms leading to the development of cervical cancer. Knowledge about HPV vaccination was piecemeal and distorted. The knowledge inadequacy had been expected and was similar to findings from other countries. ${ }^{611}$ The perceived risk of HPV infection and cervical cancer was understandably low because most participants were sexually inexperienced and did not plan to engage in sex in the near future.

Participants have a generally favourable attitude towards HPV vaccination. After the focus group, knowledge and attitude scores, as well as the proportion of participants likely to be vaccinated increased significantly, but no direct correlation between knowledge and vaccination intention was found. The small sample size of the study may have contributed to the negative findings. The lack of immediate personal relevance of cervical cancer and low intention to engage in sex in the near future may override any other influences on vaccination intention. The influence of knowledge on behavioural intention is complex and inconclusive. What was presented and how it

Table 3 Comparison of knowledge, attitudes, vaccination intention and willingness to conform before and after the focus group

\begin{tabular}{|c|c|c|c|}
\hline & Before group & After group & p Value \\
\hline Knowledge: mean (SD) & $7.94(2.26)$ & $12.81(1.39)$ & $<0.001^{*}$ \\
\hline \multirow[t]{2}{*}{ Attitudes: mean (SD) } & $20.31(4.79)$ & $21.53(3.84)$ & $0.011^{*}$ \\
\hline & $\mathrm{n}(\%)$ & $\mathrm{n}(\%)$ & \\
\hline Intention to be vaccinated now & & & $0.013 \dagger$ \\
\hline Very likely & $2(3)$ & $3(5)$ & \\
\hline Likely & $29(48)$ & $41(64)$ & \\
\hline Unlikely & $26(43)$ & $18(28)$ & \\
\hline Very unlikely & $4(7)$ & $2(3)$ & \\
\hline Willingness to conform to family & & & $0.001 \dagger$ \\
\hline Very willing & $3(5)$ & $9(14)$ & \\
\hline Willing & $43(72)$ & $51(80)$ & \\
\hline Unwilling & $11(18)$ & $2(3)$ & \\
\hline Very unwilling & $3(5)$ & $2(3)$ & \\
\hline Willingness to conform to peers & & & $0.025 \dagger$ \\
\hline Very willing & $5(8)$ & $8(13)$ & \\
\hline Willing & $45(75)$ & $51(80)$ & \\
\hline Unwilling & $6(10)$ & $4(6)$ & \\
\hline Very unwilling & $4(7)$ & $1(2)$ & \\
\hline
\end{tabular}

${ }^{*}$ Obtained by paired $\mathrm{t}$ test. †Obtained by Wilcoxon signed ranks test. 
was presented by the facilitators could potentially bias participants' response towards vaccination. It is also plausible that authoritative knowledge may have worked with other factors to potentiate the participants' acceptability of vaccination. This is especially so in a group setting, as in our study, in which favourable peer responses and group pressure may prompt the participants to accommodate to the group norm.

Positive endorsement from significant others and medical professionals were noted to be facilitating factors to enhance vaccine acceptability. The influence of others was notably strong, as demonstrated by the fact that there were proportionately more participants who were willing to conform to peers or families than their own stated intention to be vaccinated. This finding is consistent with the literature noting the strong impact of peer and parental influences on adolescent sexual behaviours. ${ }^{12}$ It is also likely that perceived negative family endorsement of HPV vaccination may have an even stronger influence than positive endorsement, particularly in view of the fact that the teenage girls are still financially dependent.

Cost is a practical issue, which prompted the participants to consider more stringently the right timing of vaccination, attempting to match the current known duration of effectiveness of the HPV vaccine with their expected age of sexual debut, which was relatively conservative in comparison with data from the west. ${ }^{13}$ Such a consideration reflected the participants' belief that sex was a planned and highly controllable act. This belief, however, is challenged by findings indicating that adolescent health-risk behaviour, such as engaging in unprotected sex, is often unplanned and unintentional. ${ }^{14}$

Of interest to note was the anticipated family disapproval against HPV vaccination by some participants. This was in contrast to findings from another qualitative study in which Chinese women were in favour of vaccinating their teenage daughters but were worried that they could not persuade the teenagers to be vaccinated. ${ }^{15}$ The potential encouragement of early sex or promiscuity as a result of HPV vaccination was not a significant concern among the mothers. To promote better acceptability in the adolescent population, however, explicit downplaying of any suspicion of sexual impropriety needs to be communicated.

Box 3: Perceptions and factors associated with the acceptability of HPV vaccination among the participants

General attitude

- Favourable with varied views on the timing of vaccination

Barriers

- High monetary cost

- Uncertain length of vaccine effectiveness

- Low perceived risk of HPV infection because not engaging in sex at present

- No immediate perceived need because of slim chance of having sex in the near future

- Anticipated family disapproval

- Fear of the pain of injection

Facilitators

- Family support

- Peer support

- Medical reassurance on safety and efficacy of vaccine.

\section{Key messages}

- Adolescent girls' knowledge about cervical cancer, HPV infection and HPV vaccination was grossly inadequate and misconceptions abound.

- HPV vaccination was viewed favourably by most of the girls. In general, however, there was no immediate perceived need for HPV vaccination as a consequence of the monetary cost, the uncertain duration of effectiveness of the vaccines and their not planning to engage in sex in the near future. The perceived timing of vaccination was largely determined by participant's intended age of sexual debut.

- Focus can be directed towards developing educational and behavioural interventions for adolescents to guard against unplanned and unprotected sex and to increase their perceived need to take preventive measures, including vaccination, against HPV or other sexually transmitted infections.

\section{Implications}

The findings indicate that there is a need to provide adolescent girls with authoritative information on cervical cancer, HPV infection and HPV vaccination to increase their understanding of why action needs to be taken at their young age to prevent a seemingly adult disease that would not happen in the immediate future. Peer and family influences are important factors affecting adolescents' attitudes towards HPV vaccination. The promotion of HPV vaccination may thus need to target both parents and the adolescent social norm. Furthermore, strategies to foster communication between parents and adolescents on the issue of HPV vaccination may help to clear up potential misunderstandings and enhance vaccine uptake.

At present, little can be done to overcome the barriers of cost and the uncertain duration of effectiveness of the vaccine on an individual level. Educational and behavioural interventions may, however, prompt adolescents to exercise caution against unplanned and unprotected sex and increase their perceived need to take preventive measures, including vaccination, against HPV or other sexually transmitted infections. ${ }^{12}$ Indeed, a better awareness of the potential hazards of unprotected sex may even discourage sexual experimentation in the youth population. On the public health level, although it has been recommended that young adolescents should be vaccinated at the age of 12 years or younger based on the expected age of sexual debut in the west, ${ }^{16}$ the situation in Asia may be different as a result of a later onset age of sex in general. ${ }^{17}$ Therefore, in recommending an optimal timing for HPV vaccination, there needs to be a realistic consideration of individual and sociocultural differences in sexual attitudes and behaviours, the preparedness for sexual ventures and socioeconomic issues.

The qualitative nature of the study in which quantification and representativeness were not the objectives inevitably imposes limitations on the findings. In particular, the sample size was small, the participants were not meant to be representative and no causal inferences could be established Despite this, the findings provide a good basis for further largescale studies of a more quantitative nature to be planned.

Acknowledgements: The authors would like to thank the Aberdeen Kai-fong Welfare Association Social Service Centre and Hon Wah College for their assistance in participant recruitment.

Funding: This research was jointly funded by the Wong Check She Charitable Foundation and the Research Fund from the Department of Obstetrics and Gynaecology, The University of Hong Kong. 


\section{Competing interests: None declared.}

Ethics approval: Before commencement of the study, approval from the Ethics Committee of the Joint Board of The University of Hong Kong and Queen Mary Hospital was obtained.

Contributors: TK, design, implementation, facilitator, analysis, chief writer of the manuscript; KC, conception, design, co-editor; AY, facilitator; KT, design, co-editor; $\mathrm{AC}$, design, co-editor; PY, facilitator; PL, project supervisor, facilitator, analysis, cowriter, co-editor; HN, research team leader, design, co-editor; TK and KC are co-first authors; HN and PL are senior and co-corresponding authors.

\section{REFERENCES}

1. Department of Health. Cervical screening: facts and figures. 1 September 2006. http://www.cervicalscreening.gov.hk/english/cc/cc_facts/cc_facts.php (accessed 5 Feb 2007).

2. Villa LL, Costa RL, Petta CA, et al. Prophylactic quadrivalent human papillomavirus (types 6, 11, 16, and 18) L1 virus-like particle vaccine in young women: a randomised double-blind placebo-controlled multicentre phase II efficacy trial. Lancet Oncol 2005;6:271-8.

3. Chan PK, Chang AR, Cheung JL, et al. Determinants of cervical human papillomavirus infection: differences between high- and low-oncogenic risk types. $J$ Infect Dis 2002;185:28-35.

4. Kitchener CR, Thompson C. Trends in sexual behaviour and safer sex perceptions in Europe, USA, Africa and Asia relating to sexual health, AIDS andcontraception. International Conference on AIDS; 7-12 July 1996, Vancouver, Canada.

5. Task Force on Youth Sexuality Study 2006 (Hong Kong). FPAHK Youth Sexuality Study 2006. http://www.famplan.org.hk/fpahk/en/info/publications/YSS2006.ppt (accessed 10 Jan 2008).
6. Tiro JA, Meissner HI, Kobrin S, et al. What do women in the U.S. know about human papillomavirus and cervical cancer? Cancer Epidemiol Biomarkers Prev 2007;16:288-94.

7. Cuschieri KS, Horne AW, Szarewski A, et al. Public awareness of human papillomavirus. J Med Screen 2006;13:201-7.

8. Ajzen I, Fishbein M. Understanding attitudes and predicting social behavior. Englewood Cliffs, NJ: Prentice-Hall, Inc, 1980.

9. Ezzy D. Qualitative analysis. London: Routledge, 2002.

10. Krueger R. Analyzing and reporting focus group results. Thousand Oaks, CA: Sage Publications, 1998.

11. Marlow LA, Waller J, Wardle J. Public awareness that HPV is a risk factor for cervical cancer. Br J Cancer 2007;97:691-4.

12. Pedlow CT, Carey MP. Developmentally appropriate sexual risk reduction interventions for adolescents: rationale, review of interventions, and recommendations for research and practice. Ann Behav Med 2004;27:172-84.

13. Abma JC, Sonenstein FL. Sexual activity and contraceptive practices among teenagers in the United States, 1988 and 1995. National Center for Health Statistics. Vital Health Stat 23 2001;21:1-79.

14. Gibbons FX, Gerrard M, Blanton H, et al. Reasoned action and social reaction: willingness and intention as independent predictors of health risk. J Pers Soc Psychol 1998; 74:1164-80.

15. Lee PW, Kwan TT, Tam KF, et al. Beliefs about cervical cancer and human papillomavirus (HPV) and acceptability of HPV vaccination among Chinese women in Hong Kong. Prev Med 2007;45:130-4.

16. Markowitz LE, Dunne EF, Saraiya M, et al. Quadrivalent human papillomavirus vaccine: recommendations of the Advisory Committee on Immunization Practices (ACIP). MMWR Recomm Rep 2007:56:1-24.

17. World Health Organization. Sexual relations among young people in developing countries: evidence from WHO case studies. Geneva: WHO, 2001.

\section{Let us assist you in teaching the next generation}

Figures from all articles on our website can be downloaded as a PowerPoint slide. This feature is ideal for teaching and saves you valuable time. Just click on the image you need and choose the "PowerPoint Slide for Teaching" option. Save the slide to your hard drive and it is ready to go. This innovative function is an important aid to any clinician, and is completely free to subscribers. (Usual copyright conditions apply.) 